\title{
WEAKLY SEQUENTIALLY CONTINUOUS MAPS AND EXISTENCE PRINCIPLES FOR ELLIPTIC EQUATIONS
}

Abstract. We present a Furi-Pera type theorem for weakly sequentially continuous maps. As an application we establish new existence principles for elliptic Dirichlet problems.

1. Introduction. In Section 2 we present new fixed point results for weakly sequentially continuous maps. In particular we extend results in $[2,5$, 12] and we also obtain a Furi-Pera type theorem [9] for weakly sequentially continuous maps in separable reflexive Banach spaces. A variation of this result can be found in [12] (where one of the conditions was stated incorrectly but applied correctly). In Section 3 we show how the results in Section 2 can be used to obtain existence principles for the elliptic Dirichlet problem

$$
\begin{cases}\Delta y+f(t, y)=0 & \text { on } \Omega \\ y=0 & \text { on } \partial \Omega\end{cases}
$$

here $\Omega$ will be a bounded domain in $\mathbb{R}^{n}, n \geq 3$, with a $C^{1,1}$ boundary $\partial \Omega$. In this paper we are interested in strong solutions to (1.1) and our results extend and complement those in $[3,4,11]$. In $[3,4,11]$ a fixed point theorem [2] for self-maps is used to obtain existence results for (1.1) and as a result some restrictions have to be placed on $f$ in order to guarantee that the appropriate operator maps a particular convex set back into itself. However using the fixed point theory in Section 2 we are able to remove this restriction so a more general result can be formulated.

2000 Mathematics Subject Classification: 47A10, 35J65.

Key words and phrases: weakly sequentially continuous, Furi-Pera type theorem, elliptic Dirichlet problem, existence principle, strong solution. 
For notational purposes $[1,10]$ for a nonnegative integer $k$ and a real number $p \in(n / 2, \infty)$ we denote by $W^{k, p}(\Omega)$ the space of all real-valued functions defined on $\Omega$ whose weak partial derivatives up to order $k$ lie in $L^{p}(\Omega)$, equipped with the usual norm. $W_{0}^{1, p}(\Omega)$ stands for the closure of $C_{0}^{\infty}(\Omega)$ in the space $W^{1, p}(\Omega)$. Also in Section $3,|\cdot|_{L^{p}}$ denotes the usual $L^{p}$ norm.

2. Fixed point theory. In this section we present the fixed point theory which will be needed in Section 3. First we state a fixed point result due to Arino, Gautier and Penot [2].

TheOREM 2.1. Let $E$ be a metrizable locally convex linear topological space and let $C$ be a weakly compact, convex subset of $E$. Then any weakly sequentially continuous map $F: C \rightarrow C$ has a fixed point.

Our next result replaces the weak compactness of the space $C$ with a weak compactness assumption on the operator $F$.

Theorem 2.2. Let $E$ be a Banach space with $C$ a closed, convex subset of $E$. Then any weakly compact, weakly sequentially continuous map $F$ : $C \rightarrow C$ has a fixed point.

Proof. There exists a weakly compact subset $K$ of $C$ with $F(C) \subseteq K \subseteq C$. The Krein-Šmulian theorem [6, p. 434] guarantees that $\overline{\mathrm{co}}(K)$ is weakly compact. Notice also that $F: \overline{\mathrm{co}}(K) \rightarrow \overline{\mathrm{co}}(K)$, so Theorem 2.1 guarantees that there exists $x \in \overline{\mathrm{co}}(K)$ with $x=F(x)$.

REMARK 2.1. In Theorem 2.2, $E$ Banach can be replaced by any metrizable locally convex linear topological space where the Krein-Smulian theorem holds; for examples see [7, p. 553; 8, p. 82].

In applications, to construct a set $C$ so that $F$ takes $C$ back into $C$ is very difficult and sometimes impossible. As a result it makes sense to discuss maps $F: C \rightarrow E$. Our first result in this direction is the so called nonlinear alternative of Leray-Schauder.

In the proof we will need the following well known result [2].

TheOREM 2.3. Let $E$ be a metrizable locally convex linear topological space with $D$ a weakly compact subset of $E$. If $F: D \rightarrow E$ is a weakly sequentially continuous map, then $F: D \rightarrow E$ is a weakly continuous map.

Theorem 2.4. Let $E$ be a Banach space, $C$ a closed convex subset of $E, U$ a weakly open subset of $C, 0 \in U$ and $\overline{U^{w}}$ weakly compact (here $\overline{U^{w}}$ denotes the weak closure of $U$ in $C$ ). Suppose $F: \overline{U^{w}} \rightarrow C$ is a weakly sequentially continuous map with the following property:

$$
x \neq \lambda F x \quad \text { for every } x \in \partial U \text { and } \lambda \in(0,1) ;
$$


here $\partial U$ denotes the weak boundary of $U$ in $C$. Then $F$ has a fixed point in $\overline{U^{w}}$.

Proof. Suppose $F$ does not have a fixed point in $\partial U$ (otherwise we are finished), so $x \neq \lambda F x$ for every $x \in \partial U$ and $\lambda \in[0,1]$. Consider

$$
A=\left\{x \in \overline{U^{w}}: x=t F(x) \text { for some } t \in[0,1]\right\} .
$$

Now $A \neq \emptyset$ since $0 \in U$. Also Theorem 2.3 guarantees that $F: \overline{U^{w}} \rightarrow C$ is weakly continuous. Thus $A$ is weakly closed and in fact weakly compact since $\overline{U^{w}}$ is weakly compact.

Also $A \cap \partial U=\emptyset$ so there exists (since $(E, w)$, the space $E$ endowed with the weak topology, is completely regular) a weakly continuous map $\mu: \overline{U^{w}} \rightarrow[0,1]$ with $\mu(\partial U)=0$ and $\mu(A)=1$. Let

$$
J(x)= \begin{cases}\mu(x) F(x), & x \in \overline{U^{w}} \\ 0, & x \in C \backslash \overline{U^{w}} .\end{cases}
$$

Clearly $J: C \rightarrow C$ is a weakly compact, weakly sequentially continuous map. Theorem 2.2 guarantees that there exists $x \in C$ with $x=J(x)$. Notice that $x \in U$ since $0 \in U$. As a result $x=\mu(x) F(x)$, so $x \in A$. Thus $\mu(x)=1$ and so $x=F(x)$.

Next we present a Furi-Pera theorem for weakly sequentially continuous maps. This result can be found in [12]; we note that one of the conditions there is stated incorrectly and that the proof there has to be adjusted slightly.

TheOREm 2.5. Let $E$ be a separable and reflexive Banach space, and let $C$ and $Q$ be closed bounded convex subsets of $E$ with $Q \subseteq C$ and $0 \in Q$. Suppose $F: Q \rightarrow C$ is a weakly sequentially continuous map and assume the following condition is satisfied:

if $\left\{\left(x_{j}, \lambda_{j}\right)\right\}_{j=1}^{\infty}$ is a sequence in $Q \times[0,1]$ with $x_{j} \rightarrow x \in \partial Q$ and $\lambda_{j} \rightarrow \lambda$, and if $x=\lambda F(x)$ for $0 \leq \lambda<1$, then there exists $j_{0} \in$ $\{1,2, \ldots\}$ with $\lambda_{j_{0}} F\left(x_{j_{0}}\right) \in Q$; here $\partial Q$ denotes the weak boundary of $Q$ relative to $C$ and $\rightarrow$ denotes weak convergence.

Then $F$ has a fixed point in $Q$.

Remark 2.2. A special case of (2.2) (which is all we need in Section 3) is the following condition:

if $\left\{\left(x_{j}, \lambda_{j}\right)\right\}_{j=1}^{\infty}$ is a sequence in $Q \times[0,1]$ with $x_{j} \rightarrow x$ and $\lambda_{j} \rightarrow \lambda$, and if $x=\lambda F(x)$ for $0 \leq \lambda<1$, then there exists $j_{0} \in\{1,2, \ldots\}$ with $\lambda_{j_{0}} F\left(x_{j_{0}}\right) \in Q$.

Proof of Theorem 2.5. Let $r: E \rightarrow Q$ be a weakly continuous retraction (see [12]) and let

$$
B=\{x \in E: x=\operatorname{Fr}(x)\} .
$$


Note that $B \subseteq C$ since $F: Q \rightarrow C$. It is easy to see that $B \neq \emptyset$ is weakly closed and weakly compact (note that $C$ is weakly compact since $C$ is closed and convex (so weakly closed) and bounded in the norm topology). It remains to show $B \cap Q \neq \emptyset$. Suppose $B \cap Q=\emptyset$. Also since $E$ is separable we know from [6] that the weak topology on $C$ is metrizable; let $d^{\star}$ denote the metric. With respect to $\left(C, d^{\star}\right)$ note that $Q$ is closed, $B$ is compact, $B \cap Q=\emptyset$ so there exists $\varepsilon>0$ with

$$
d^{\star}(B, Q)=\inf \left\{d^{\star}(x, y): x \in B, y \in Q\right\}>\varepsilon .
$$

For $i \in\{1,2, \ldots\}$ let

$$
U_{i}=\left\{x \in C: d^{\star}(x, Q)<\varepsilon / i\right\} .
$$

Fix $i \in\{1,2, \ldots\}$. Now $U_{i}$ is $d^{\star}$-open in $C$, so $U_{i}$ is weakly open in $C$. Also

$$
\overline{U_{i}^{w}}=\overline{U_{i}^{d^{\star}}}=\left\{x \in C: d^{\star}(x, Q) \leq \varepsilon / i\right\}, \quad \partial U_{i}=\left\{x \in C: d^{\star}(x, Q)=\varepsilon / i\right\} .
$$

Now $B \cap \overline{U_{i}^{w}}=\emptyset$ (since $d^{\star}(B, Q)>\varepsilon$ ) and Theorem 2.4 (with $F=F r$ and $\left.U=U_{i}\right)$ guarantees that there exist $\lambda_{i} \in(0,1)$ and $y_{i} \in \partial U_{i}$ with $y_{i}=\lambda_{i} \operatorname{Fr}\left(y_{i}\right)$. We can do this argument for each $i \in\{1,2, \ldots\}$. Notice in particular since $y_{i} \in \partial U_{i}$ that

$$
\lambda_{i} \operatorname{Fr}\left(y_{i}\right) \notin Q \quad \text { for each } i \in\{1,2, \ldots\} .
$$

Now look at

$$
D=\{x \in E: x=\lambda F r(x) \text { for some } \lambda \in[0,1]\} .
$$

Since $D$ is weakly compact (so weakly sequentially compact by the EberleinŠmulian theorem) and

$$
d^{\star}\left(y_{j}, Q\right)=\varepsilon / j, \quad\left|\lambda_{j}\right| \leq 1 \quad \text { for } j \in\{1,2, \ldots\},
$$

we may assume without loss of generality that

$$
\lambda_{j} \rightarrow \lambda^{\star}, \quad y_{j} \rightarrow y^{\star} \in \overline{Q^{w}} \cap \overline{C \backslash Q^{w}}=\partial Q .
$$

Also since $y_{j}=\lambda_{j} \operatorname{Fr}\left(y_{j}\right)$ we have $y^{\star}=\lambda^{\star} \operatorname{Fr}\left(y^{\star}\right)$ (recall $\mathrm{Fr}: C \rightarrow C$ is weakly continuous). If $\lambda^{\star}=1$ then $y^{\star}=F r\left(y^{\star}\right)$, which contradicts $B \cap Q=\emptyset$. Thus $0 \leq \lambda^{\star}<1$. But in this case $(2.2)$, with

$$
x_{j}=r\left(y_{j}\right) \text { and } \quad x=y^{\star}=r\left(y^{\star}\right),
$$

implies there exists $j_{0} \in\{1,2, \ldots\}$ with $\lambda_{j_{0}} \operatorname{Fr}\left(y_{j_{0}}\right) \in Q$. This contradicts (2.4). Thus $B \cap Q \neq \emptyset$. As a result there exists $x \in Q$ with $x=\operatorname{Fr}(x)=$ $F(x)$.

3. Applications. In this section we present two existence principles for strong solutions to

$$
\begin{cases}\Delta y+f(t, y)=0 & \text { on } \Omega \\ y=0 & \text { on } \partial \Omega\end{cases}
$$


Our results improve those in [13]; we note that the set $Q$ in [13] was chosen incorrectly so the argument in [13] has to be adjusted slightly as indicated in this paper.

Throughout this section $\Omega$ will be a bounded domain in $\mathbb{R}^{n}, n \geq 3$, with a $C^{1,1}$ boundary $\partial \Omega$. For our first two results $f: \Omega \times \mathbb{R} \rightarrow \mathbb{R}$ will be an $L^{\infty}$-Carathéodory function; by this we mean

(a) $t \mapsto f(t, x)$ is measurable for every $x \in \mathbb{R}$,

(b) $x \mapsto f(t, x)$ is continuous for a.e. $t \in \Omega$,

(c) for each $r>0$, there exists $h_{r} \in L^{\infty}(\Omega)$ with $|f(t, x)| \leq h_{r}(t)$ for a.e. $t \in \Omega$ and every $x \in \mathbb{R}$ with $|x| \leq r$.

Also for our first two results let $q \in(n / 2, \infty)$ be fixed and let

$$
X_{\infty}(\Omega)=\left\{u \in W^{2, q}(\Omega) \cap W_{0}^{1, q}(\Omega): \Delta u \in L^{\infty}(\Omega)\right\} .
$$

A function $y: \Omega \rightarrow \mathbb{R}$ is said to be a strong solution to (3.1) (in the $L^{\infty}$ sense) if $y \in X_{\infty}(\Omega)$ with $\Delta y+f(t, y)=0$ for a.e. $t \in \Omega$ and $y$ satisfies the boundary condition.

Our existence principles for (3.1) will be based on Theorem 2.5, and they improve results in $[3,4,11]$.

Theorem 3.1. Let $f: \Omega \times \mathbb{R} \rightarrow \mathbb{R}$ be an $L^{\infty}$-Carathéodory function and assume there exists a constant $M_{0}$ (independent of $\lambda$ ) with $|\Delta y|_{\infty} \leq M_{0}$ for any solution $y \in X_{\infty}(\Omega)$ to

$$
\begin{cases}\Delta y+\lambda f(t, y)=0 & \text { on } \Omega, \\ y=0 & \text { on } \partial \Omega,\end{cases}
$$

for $0<\lambda<1$. Then (3.1) has at least one strong solution.

Proof. Let

$$
A_{0}= \begin{cases}\frac{1}{2 n \pi}[\Gamma(1+n / 2)|\Omega|]^{2 / n} & \text { if }|\Omega|>1, \\ \frac{1}{2 n \pi}[\Gamma(1+n / 2)]^{2 / n} & \text { if }|\Omega| \leq 1,\end{cases}
$$

where $|\Omega|$ is the Lebesgue measure of $\Omega$ and $\Gamma$ is the Gamma function. Since $f$ is $L^{\infty}$-Carathéodory, there exists $h_{A_{0}\left(M_{0}+1\right)} \in L^{\infty}(\Omega)$ with

$$
\begin{aligned}
& |f(t, u)| \leq h_{A_{0}\left(M_{0}+1\right)}(t) \text { for a.e. } t \in \Omega \text { and every } u \in \mathbb{R} \text { with }|u| \leq \\
& A_{0}\left(M_{0}+1\right) .
\end{aligned}
$$

We will apply Theorem 2.5 with $E=L^{q}(\Omega)$,

$$
\begin{aligned}
& Q=\left\{u \in L^{q}(\Omega): u \in L^{\infty}(\Omega) \text { and }|u|_{\infty} \leq M_{0}+1\right\}, \\
& C=\left\{u \in L^{q}(\Omega):|u(t)| \leq \phi(t) \text { for a.e. } t \in \Omega\right\},
\end{aligned}
$$

where $\phi(t)=\max \left\{M_{0}+1, h_{A_{0}\left(M_{0}+1\right)}(t)\right\}$. 
First note that $Q$ and $C$ are bounded, convex subsets of $E$. Next we show $Q$ is closed. To see this let $u_{n} \in Q(n \in \mathbb{N}=\{1,2, \ldots\})$ with $u_{n} \rightarrow u$ in $L^{q}(\Omega)$. This implies $\left|u_{n}(t)\right| \leq M_{0}+1$ for a.e. $t \in \Omega$ for each $n \in \mathbb{N}$. In addition since $u_{n} \rightarrow u$ in $L^{q}(\Omega)$ there exists a subsequence $S$ of $\mathbb{N}$ with

$$
u_{n}(t) \rightarrow u(t) \quad \text { for a.e. } t \in \Omega \text { as } n \rightarrow \infty \text { in } S \text {. }
$$

Consequently, $|u(t)| \leq M_{0}+1$ for a.e. $t \in \Omega$, so $|u|_{\infty} \leq M_{0}+1$. Thus $x \in Q$, so $Q$ is closed. Similarly $C$ is closed. Note also that $Q \subseteq C$ and $0 \in Q$.

Let $\psi: X_{\infty}(\Omega) \rightarrow L^{\infty}(\Omega)$ be defined by $\psi(u)=-\Delta u$. Now [10, Theorem 9.15 , p. 241] guarantees that $\psi$ is a one-to-one mapping from $W^{2, q}(\Omega) \cap$ $W_{0}^{1, q}(\Omega)$ onto $L^{q}(\Omega)$. For $u \in Q$ and a.e. $t \in \Omega$ let

$$
F u(t)=f\left(t, \psi^{-1}(u)(t)\right) .
$$

We will use Theorem 2.5 to show $F$ has a fixed point in $Q$. Of course we need to check that $F: Q \rightarrow C$ is weakly sequentially continuous and that (2.3) holds.

First we show $F: Q \rightarrow C$. If $y \in Q$ then $|y|_{\infty} \leq M_{0}+1$ and this together with [3, p. 32,15] implies for a.e. $s \in \Omega$ that

$$
\left|\psi^{-1}(y(s))\right| \leq\left|\psi^{-1}(y)\right|_{\infty} \leq A_{0}|y|_{\infty} \leq A_{0}\left(M_{0}+1\right) .
$$

This together with (3.2) gives

$$
|F y(s)|=\left|f\left(s, \psi^{-1}(y)(s)\right)\right| \leq h_{A_{0}\left(M_{0}+1\right)}(s) \quad \text { for a.e. } s \in \Omega,
$$

and so $F y \in C$. Thus $F: Q \rightarrow C$.

To show $F: Q \rightarrow C$ is weakly sequentially continuous, let $x_{n} \in Q$, $n \in \mathbb{N}$, with $x_{n} \rightarrow x$ in $L^{q}(\Omega)$. Now [10, Lemma 9.17 , p. 242] guarantees that $\psi^{-1}$ is a continuous linear operator from $L^{q}(\Omega)$ into $W^{2, q}(\Omega)$ (so weakly continuous), and so we have $\psi^{-1}\left(x_{n}\right) \rightarrow \psi^{-1}(x)$ in $W^{2, q}(\Omega)$. The RellichKondrashov theorem $[1$, Theorem 6.2 , p. 144] guarantees (note $q>n / 2$ ) that the imbedding $W^{2, q}(\Omega) \rightarrow C(\bar{\Omega})$ is completely continuous, so there exists a subsequence $S$ of $\mathbb{N}$ with

$$
\psi^{-1}\left(x_{n}\right) \rightarrow \psi^{-1}(x) \quad \text { in } C(\bar{\Omega}) \text { as } n \rightarrow \infty \text { in } S .
$$

Now $f$ is $L^{\infty}$-Carathéodory, so

$$
F x_{n}(t) \rightarrow F x(t) \quad \text { a.e. in } \Omega \text { as } n \rightarrow \infty \text { in } S,
$$

and

$$
\left|F x_{n}(t)\right| \leq \phi(t) \quad \text { for a.e. } t \in \Omega .
$$

The Lebesgue dominated convergence theorem implies

$$
\lim _{n \rightarrow \infty} F x_{n}=F x \quad \text { in } L^{q}(\Omega),
$$

so $F x_{n} \rightarrow F x$ in $L^{q}(\Omega)$. Thus $F: Q \rightarrow C$ is weakly sequentially continuous. 
It now remains to check (2.3). Take a sequence $\left\{\left(x_{j}, \lambda_{j}\right)\right\}_{j=1}^{\infty}$ in $Q \times[0,1]$ with $\lambda_{j} \rightarrow \lambda$ and $x_{j} \rightarrow x$ with $x=\lambda F x, 0 \leq \lambda<1$. Since $x_{j} \rightarrow x$, as in (3.3) there exists a subsequence $S$ of $\mathbb{N}$ with $F x_{j} \rightarrow F x$ in $L^{q}(\Omega)$ as $j \rightarrow \infty$ in $S$. Thus there exists a subsequence $S_{1}$ of $S$ with

$$
F x_{j}(t) \rightarrow F x(t) \quad \text { for a.e. } t \in \Omega \text { as } j \rightarrow \infty \text { in } S_{1} .
$$

Now Egorov's theorem [14, p. 975] implies

$$
F x_{j} \rightarrow F x \quad \text { almost uniformly on } \Omega \text { as } j \rightarrow \infty \text { in } S_{1} .
$$

Since $x=\lambda F x$, we see that $u=\psi^{-1}(x)$ satisfies $\Delta u+\lambda f(t, u)=0$ a.e. on $\Omega$ and $u=0$ on $\partial \Omega$. By hypothesis, $|\Delta u|_{\infty} \leq M_{0}$ and so $|x|_{\infty} \leq M_{0}$. Now given $\varepsilon>0$ (say $\varepsilon<1 / 3$ ) we know from (3.4) that there exists $j_{0} \in S_{1}$ with

$$
\left|F x_{j}(t)\right| \leq|F x(t)|+\varepsilon \quad \text { for } j \geq j_{0}\left(j \in S_{1}\right) \text { for a.e. } t \in \Omega .
$$

As a result for a.e. $t \in \Omega$ and $j \geq j_{0}\left(j \in S_{1}\right)$ we have (note that $Q$ is weakly closed, so $x \in Q$ and $F x \in C$ since $x_{j} \rightarrow x$ )

$$
\begin{aligned}
\left|\lambda_{j} F x_{j}(t)\right| & \leq\left|\lambda_{j}-\lambda\right||F x(t)|+|\lambda F x(t)|+\varepsilon \\
& \leq\left|\lambda_{j}-\lambda\right| \phi(t)+|x(t)|+\varepsilon \leq\left|\lambda_{j}-\lambda\right||\phi|_{\infty}+M_{0}+\varepsilon .
\end{aligned}
$$

Now since $\lambda_{j} \rightarrow \lambda$ there exists $j_{1} \in S_{1}\left(j_{1} \geq j_{0}\right)$ with

$$
\left|\lambda_{j} F x_{j}(t)\right| \leq M_{0}+1 \quad \text { for a.e. } t \in \Omega\left(j \geq j_{1} \text { and } j \in S_{1}\right),
$$

and so

$$
\left|\lambda_{j} F x_{j}\right|_{\infty} \leq M_{0}+1 \quad \text { for } j \geq j_{1}\left(j \in S_{1}\right) .
$$

As a result $\lambda_{j} F x_{j} \in Q$ for $j \in S_{1}$ sufficiently large, so (2.3) holds. Now Theorem 2.5 guarantees that there exists $u \in Q$ with $u=F u$. Then the function $y(t)=\psi^{-1}(u)(t), t \in \Omega$, is a strong solution to (3.1).

REMARK 3.1. In the proof of Theorem 3.1 we could have taken $C$ to be

$$
\left\{u \in L^{q}(\Omega): u \in L^{\infty}(\Omega) \text { and }|u|_{\infty} \leq M_{1}\right\}
$$

where $M_{1}=\max \left\{M_{0}+1,\left|h_{A_{0}\left(M_{0}+1\right)}\right|_{\infty}\right\}$.

Remark 3.2. The ideas in Theorem 3.1 (the details are left to the reader) extend to the problem

$$
\begin{cases}L y+f(t, y)=0 & \text { on } \Omega, \\ y=0 & \text { on } \partial \Omega,\end{cases}
$$

where $L$ is a linear second order elliptic differential operator.

Our final existence result concerns the case when $f: \Omega \times \mathbb{R} \rightarrow \mathbb{R}$ is an $L^{p}$-Carathéodory function (here $\left.p \in(n / 2, \infty)\right)$; by this we mean

(a) $t \mapsto f(t, x)$ is measurable for every $x \in \mathbb{R}$,

(b) $x \mapsto f(t, x)$ is continuous for a.e. $t \in \Omega$, 
(c) for each $r>0$, there exists $h_{r} \in L^{p}(\Omega)$ with $|f(t, x)| \leq h_{r}(t)$ for a.e. $t \in \Omega$ and every $x \in \mathbb{R}$ with $|x| \leq r$.

Let $X_{p}(\Omega)=W^{2, p}(\Omega) \cap W_{0}^{1, p}(\Omega)$. A function $y: \Omega \rightarrow \mathbb{R}$ is said to be a strong solution to (3.1) (in the $L^{p}$ sense) if $y \in X_{p}(\Omega)$ with $\Delta y+f(t, y)=0$ for a.e. $t \in \Omega$ and $y$ satisfies the boundary condition.

We use Theorem 2.5 to prove our next result. We could also use the usual Leray-Schauder alternative (with the strong topology). When one uses the strong topology one needs to check the compactness of the map (this is currently easy since there are many available results in the literature). However, in our opinion, the weakly sequentially continuous approach is easier and quicker since one does not need to check the compactness of the map (i.e. once the appropriate results are available in the literature for weakly sequentially continuous maps the result will also be immediate).

Theorem 3.2. Let $p \in(n / 2, \infty)$ and let $f: \Omega \times \mathbb{R} \rightarrow \mathbb{R}$ be an $L^{p}$ Carathéodory function. Assume there exists a constant $M_{0}$ (independent of $\lambda)$ with $|\Delta y|_{L^{p}(\Omega)} \leq M_{0}$ for any solution $y \in X_{p}(\Omega)$ to

$$
\begin{cases}\Delta y+\lambda f(t, y)=0 & \text { on } \Omega, \\ y=0 & \text { on } \partial \Omega,\end{cases}
$$

for $0<\lambda<1$. Then (3.1) has at least one strong solution.

Proof. Let

$$
B_{0}=|\Omega|^{2 / n-1 / p} \frac{\Gamma(1+n / 2)^{2 / n}}{n(n-2) \pi}\left[\frac{\Gamma\left(1+\frac{p}{p-1}\right) \Gamma\left(\frac{n}{n-2}-\frac{p}{p-1}\right)}{\Gamma\left(\frac{n}{n-2}\right)}\right]^{1-1 / p} .
$$

Now there exists $h_{B_{0}\left(M_{0}+1\right)} \in L^{p}(\Omega)$ with

$$
\begin{aligned}
& |f(t, u)| \leq h_{B_{0}\left(M_{0}+1\right)}(t) \text { for a.e. } t \in \Omega \text { and every } u \in \mathbb{R} \text { with }|u| \leq \\
& B_{0}\left(M_{0}+1\right) .
\end{aligned}
$$

We will apply Theorem 2.5 with $E=L^{p}(\Omega)$,

$$
\begin{aligned}
& Q=\left\{u \in L^{p}(\Omega):|u|_{L^{p}(\Omega)} \leq M_{0}+1\right\}, \\
& C=\left\{u \in L^{p}(\Omega):|u(t)| \leq \phi(t) \text { for a.e. } t \in \Omega\right\},
\end{aligned}
$$

where

$$
\phi(t)=\max \left\{M_{0}+1, h_{B_{0}\left(M_{0}+1\right)}(t)\right\} .
$$

Let $\psi: X_{p}(\Omega) \rightarrow L^{p}(\Omega)$ be defined by $\psi(u)=-\Delta u$ and for $u \in Q$ and a.e. $t \in \Omega$ let

$$
F u(t)=f\left(t, \psi^{-1}(u)(t)\right) .
$$

We first show $F: Q \rightarrow C$. To see this let $y \in Q$, so $|y|_{L^{p}(\Omega)} \leq M_{0}+1$. Now $[11$, p. 60] implies for a.e. $s \in \Omega$ that

$$
\left|\psi^{-1}(y(s))\right| \leq\left|\psi^{-1}(y)\right|_{\infty} \leq B_{0}|y|_{L^{p}(\Omega)} \leq B_{0}\left(M_{0}+1\right),
$$


SO

$$
|F y(s)| \leq h_{B_{0}\left(M_{0}+1\right)}(s) \quad \text { for a.e. } s \in \Omega .
$$

Thus $F y \in C$, so $F: Q \rightarrow C$.

To see that $F: Q \rightarrow C$ is weakly sequentially continuous, let $x_{n} \in Q$, $n \in \mathbb{N}$, with $x_{n} \rightarrow x$ in $L^{p}(\Omega)$. As in Theorem 3.1 there exists a subsequence $S$ of $\mathbb{N}$ with

$$
\psi^{-1}\left(x_{n}\right) \rightarrow \psi^{-1}(x) \quad \text { in } C(\bar{\Omega}) \text { as } n \rightarrow \infty \text { in } S .
$$

Thus

$$
F x_{n}(t) \rightarrow F x(t) \quad \text { a.e. in } \Omega \text { as } n \rightarrow \infty \text { in } S,
$$

and

$$
\left|F x_{n}(t)\right| \leq \phi(t) \quad \text { for a.e. } t \in \Omega .
$$

The Lebesgue dominated convergence theorem implies

$$
\lim _{n \rightarrow \infty} F x_{n}=F x \quad \text { in } L^{p}(\Omega),
$$

so $F x_{n} \rightarrow F x$ in $L^{p}(\Omega)$. Thus $F: Q \rightarrow C$ is weakly sequentially continuous.

It now remains to check (2.3). Take a sequence $\left\{\left(x_{j}, \lambda_{j}\right)\right\}_{j=1}^{\infty}$ in $Q \times[0,1]$ with $\lambda_{j} \rightarrow \lambda$ and $x_{j} \rightarrow x$ with $x=\lambda F x, 0 \leq \lambda<1$. Since $x_{j} \rightarrow x$, the argument used to prove (3.6) guarantees that there is a subsequence $S$ of $\mathbb{N}$ with $F x_{j} \rightarrow F x$ in $L^{p}(\Omega)$ as $j \rightarrow \infty$ in $S$. Now given $\varepsilon>0$ (say $\varepsilon<1 / 3$ ), there exists $j_{0} \in S$ with

$$
\left|F x_{j}\right|_{L^{p}(\Omega)} \leq|F x|_{L^{p}(\Omega)}+\varepsilon \quad \text { for } j \geq j_{0}(j \in S) .
$$

This together with $x=\lambda F x$, and so $|x|_{L^{p}(\Omega)} \leq M_{0}$, yields

$$
\begin{aligned}
\left|\lambda_{j} F x_{j}\right|_{L^{p}(\Omega)} & \leq\left|\lambda_{j}-\lambda\right||F x|_{L^{p}(\Omega)}+|x|_{L^{p}(\Omega)}+\varepsilon \\
& \leq\left|\lambda_{j}-\lambda\right||\phi|_{L^{p}(\Omega)}+M_{0}+\varepsilon
\end{aligned}
$$

for $j \geq j_{0}$ and $j \in S$. Now since $\lambda_{j} \rightarrow \lambda$ there exists $j_{1} \in S\left(j_{1} \geq j_{0}\right)$ with

$$
\left|\lambda_{j} F x_{j}\right|_{L^{p}(\Omega)} \leq M_{0}+1 \quad \text { for } j \geq j_{1} \text { and } j \in S_{1} .
$$

As a result $\lambda_{j} F x_{j} \in Q$ for $j \in S$ sufficiently large, so (2.3) holds. We may apply Theorem 2.5 .

\section{References}

[1] R. A. Adams, Sobolev Spaces, Academic Press, 1975.

[2] O. Arino, S. Gautier and J. P. Penot, A fixed point theorem for sequentially continuous mappings with applications to ordinary differential equations, Funkc. Ekvac. 27 (1984), 273-279.

[3] G. Bonanno, Existence theorems on the Dirichlet problem for the equation $\Delta u+$ $f(t, u)=0$, Proc. Edinburgh Math. Soc. 39 (1996), 31-36. 
[4] G. Bonanno and S. Marano, Positive solutions of elliptic equations with discontinuous nonlinearities, Topol. Methods Nonlinear Anal. 8 (1996), 263-273.

[5] F. E. Browder, Nonlinear Operators and Nonlinear Equations of Evolution in Banach Spaces, in: Proc. Sympos. Pure Math. 18, Amer. Math. Soc., 1976, 1-305.

[6] N. Dunford and J. T. Schwartz, Linear Operators: Part I, General Theory, Interscience, New York, 1985.

[7] R. E. Edwards, Functional Analysis, Theory and Applications, Holt, Rinehart and Winston, 1965.

[8] K. Floret, Weakly Compact Sets, Lecture Notes in Math. 801, Springer, 1980.

[9] M. Furi and M. P. Pera, A continuation method on locally convex spaces and applications to ordinary differential equations on noncompact intervals, Ann. Polon. Math. 47 (1987), 331-346.

[10] D. Gilbarg and N. S. Trudinger, Elliptic Partial Differential Equations of Second Order, Springer, Berlin, 1983.

[11] S. Marano, Existence theorems for a semilinear elliptic boundary value problem, Ann. Polon. Math. 60 (1994), 57-67.

[12] D. O'Regan, A continuation method for weakly condensing operators, Z. Anal. Anwend. 15 (1996), 565-578.

[13] - A general existence principle for strong solutions to the Dirichlet problem for the equation $\Delta u+f(t, u)=0$, Appl. Math. Lett. 10 (1997), 29-32.

[14] C. Swartz, Measure, Integration and Function Spaces, World Sci., Singapore, 1994.

[15] G. Talenti, Elliptic equations and rearrangements, Ann. Scuola Norm. Sup. Pisa Cl. Sci. 3 (1976), 697-718.

[16] K. Yosida, Functional Analysis, Springer, Berlin, 1980.

Department of Mathematical Science

Florida Institute of Technology

Melbourne, FL 32901, U.S.A.

E-mail: agarwal@fit.edu
Department of Mathematics National University of Ireland Galway, Ireland E-mail: donal.oregan@nuigalway.ie

Received on 19.9.2002; 